\title{
Postoperative patient-controlled epidural analgesia in patients with spondylodiscitis and posterior spinal fusion surgery
}

\author{
Florian Gessler, MD, ${ }^{1}$ Haitham Mutlak, MD, ${ }^{1,2}$ Karima Tizi, MD, ${ }^{1}$ Christian Senft, MD, PhD, ${ }^{1}$ \\ Matthias Setzer, MD, PhD, ${ }^{1}$ Volker Seifert, MD, PhD, ${ }^{1}$ and Lutz Weise, MD, PhD ${ }^{1}$ \\ Departments of ${ }^{1}$ Neurosurgery and ${ }^{2}$ Anesthesia, Intensive Care Medicine, and Pain Therapy, University Hospital Frankfurt, \\ Goethe-University, Frankfurt, Germany
}

\begin{abstract}
OBJECTIVE The value of postoperative epidural analgesia after major spinal surgery is well established. Thus far, the use of patient-controlled epidural analgesia (PCEA) has been denied to patients undergoing debridement and instrumentation in spondylodiscitis, with the risk of increased postoperative pain resulting in prolonged recovery. The value of PCEA with special regard to infectious complications remains to be clarified. The present study examined the value of postoperative PCEA in comparison with intravenous analgesia in patients with spondylodiscitis undergoing posterior spinal surgery.
\end{abstract}

METHODS Thirty-two patients treated surgically for spondylodiscitis of the thoracic and lumbar spine were prospectively included in a database and retrospectively reviewed for this study. Postoperative antibiotic treatment, functional capacity, pain levels, side effects, and complications were documented. Sixteen patients were given patient-demanded intravenous analgesia (PIA) followed by 16 patients assigned to PCEA. If PCEA was applied, the insertion of an epidural catheter was performed under the direct visual guidance of the surgeon at the end of the surgery.

RESULTS Three patients intended for PCEA treatment were excluded due to predefined exclusion criteria. Postoperative pain was significantly lower in the PCEA group during the first 48 hours after surgery $(p=0.03)$. As determined by the trunk control test conducted at $8(p<0.001), 24(p=0.004), 48(p=0.015), 72(p=0.0031)$, and 96 hours $(p<0.001)$, patients in the PCEA treatment group displayed significantly increased mobilization capacity compared with those of the PIA group. Time until normal accomplishment of all mobilization maneuvers was reduced in the PCEA group compared with that in the PIA group $(p=0.04)$. No differences in complication rates were observed between the 2 groups $(p=$ 0.52).

CONCLUSIONS PCEA may reduce postoperative pain and lead to earlier achievement of functional capacity at a low complication rate in patients with surgically treated lumbar and thoracic spondylodiscitis.

http://thejns.org/doi/abs/10.3171/2015.8.SPINE15415

KEY WORDS spondylodiscitis; postoperative pain; patient-controlled analgesia; epidural catheter; postoperative infection

$\mathrm{S}$ PONDYLODISCITIS is a rare disease with a reported incidence of $1: 100,000$ and $1: 250,000$ per year in Western developed countries. ${ }^{1,4}$ Spondylodiscitis is treated with debridement of the infectious mass; restoration of spinal stability, with the treatment goal of recovery from neurological deficits; and pain management therapy.

A surgical approach facilitates early mobilization of patients, which results in lower rates of decubitus ulceration ${ }^{8}$ and other sequelae of long-term bed rest such as deep vein thrombosis, pulmonary embolism, and pneumonia. ${ }^{18}$ Instrumented stabilization accompanied by debridement and titanium graft implantation is the standard surgical procedure..$^{13,19}$

As a consequence of spinal fusion surgery, major and persistent postoperative pain may lead to an abrogation of the beneficial effects observed by surgical treatment, as

ABBREVIATIONS ICU = intensive care unit; PCEA = patient-controlled epidural analgesia; $\mathrm{PIA}=$ patient-demanded intravenous analgesia; $T C T=$ trunk control test; VAS = visual analog scale.

SUBMITTED April 9, 2015. ACCEPTED August 11, 2015.

INCLUDE WHEN CITING Published online February 5, 2016; DOI: 10.3171/2015.8.SPINE15415. 
the functional rehabilitation of patients may be hindered. Especially in the first days following surgery, sufficient analgesic treatment is necessary. ${ }^{2,6}$ Epidural $^{3,5,9,10,17}$ analgesia has been shown to be sufficient in the treatment of postoperative pain after lumbar spine discectomy and fusion.

In spondylodiscitis, local and systemic inflammation are increased. Higher concentrations of inflammatory mediators such as TNF- $\alpha$, IL- $1 \beta$, and MCP-1 have been observed and may result in reduced morphine sensitivity. ${ }^{11,21}$ Local administration of analgesics by epidural catheters and patient-controlled administration may lead to beneficial effects including reduced pain levels and increased functional status; however, the insertion of an epidural catheter into a locally infectious region is highly controversial. A concurrent infection within the insertion area is considered a contraindication in the anesthesiological literature. ${ }^{14}$

After spinal stabilizing surgery, neuromuscular activation of paraspinal muscles may be affected, resulting in functional limitations in muscular strength and functional performance. ${ }^{12,20}$ In patients with lumbar back pain, altered trunk control in regard to forward bending, rising from a chair, and walking have been described..$^{15}$ As a result of these circumstances, limited back motion and decreased muscle strength may be observed in response to either the procedure itself or due to pain-related avoidance..$^{15}$

The aim of this study was to investigate whether placement of an epidural catheter is safe after surgical sanitization of the infectious focus and, furthermore, to observe the effects of patient-controlled epidural analgesia (PCEA) versus patient-demanded intravenous analgesia (PIA) on clinical and functional capabilities and to monitor side effects.

\section{Methods}

The study was approved by the local ethics committee, and informed consent was obtained from all patients prior to surgery. We retrospectively analyzed our prospectively maintained spondylodiscitis database for data consisting of sex, age, localization of the spondylodiscitis, treatment, postoperative complications, and the treatment for patients who underwent surgery via a posterior approach between January 1, 2010, and June 30, 2013. All of the patients were treated with open instrumentation; no percutaneous instrumentation was performed in any of the cases. Patients with spondylodiscitis of the cervical spine were excluded from analysis due to the possible side effects (e.g., respiratory suppression) of overdosed analgesics. Further exclusion criteria were drug addiction and intraoperative damage of the dura.

Prior to March 2012, no peridural catheter was inserted in cases in which an infectious spondylodiscitis was observed intraoperatively. Challenging this philosophy, we revised our standardized treatment regimen in terms of epidural catheter placement in March 2012, resulting in 2 cohorts: patients who underwent epidural anesthesia and those who did not.

As an institutional standard of care, all patients with surgical treatment for spondylodiscitis were postopera- tively admitted to the neurosurgical intensive care unit (ICU) and observed until postoperative Day 1. Routine postoperative CT was performed on Day 1 of surgery to document positioning of instrumentation and catheter placement in patients of the PCEA group. Postoperative care regarding thromboprophylaxis did not differ between the 2 groups.

Preoperatively, patients were given oral premedication consisting of $7.5 \mathrm{mg}$ midazolame. Anesthesia was induced intravenously with $3 \mu \mathrm{g} / \mathrm{kg}$ fentanyl, $2 \mathrm{mg} / \mathrm{kg}$ propofol, and $0.1 \mathrm{mg} / \mathrm{kg}$ cisatracurium and maintained with repetitive bolus dosing of $1-2 \mu \mathrm{g} / \mathrm{kg}$ fentanyl and inhalative 1.1 vol \% sevoflurane. After intubation, patients underwent pressure-controlled ventilation with an inspiratory oxygen fraction of 0.5 and a tidal volume of $8-10 \mathrm{ml} / \mathrm{kg}$.

Surgery was performed with the patient in a prone position, and the infected intervertebral disc material was removed to the greatest extent possible. Antibiotic-impregnated PerOssal (hydroxyapatite with calcium sulfate) was administered to the intervertebral space. Gentamicin was applied as the standard, but the antibiotics were adjusted in cases with a known pathogen and in accordance with results of an antibiogram. Stability was achieved via dorsal instrumentation, with pedicle screws inserted as anchor points and connected with a fixateur interne for transpedicular distraction and stabilization.

After instrumentation, the neurosurgeon inserted the epidural catheter (epidural mini pack, Smith Medical) in the midline in patients of the PCEA group. The catheter tip was positioned 1 segment cranial to the treated segment, and positioning was documented in the surgeon's report. Catheters were channeled distant to the incision and anchored to the skin by a suture. As institutional standard, we routinely performed early postoperative CT for the verification of adequate instrumentation localization, allowing us to verify the precise position of the catheter tip using high-resolution spiral CT imaging and multiplanar/3D reconstruction.

Postoperative analgesia was initiated in both PCEA and PIA patients on admission to the ICU. An initial $4 \mathrm{ml}$ bolus of $0.75 \mathrm{mg} / \mathrm{ml}$ ropivacaine was administered to the patients in the PCEA group. A postoperative infusion rate of $8 \mathrm{ml} / \mathrm{hr}$ of $0.2 \mathrm{mg} / \mathrm{ml}$ ropivacaine was then initiated, and patients were allowed to receive additional boluses of $8 \mathrm{mg}$ ropivacaine every 20 minutes on demand using the PCEA software. Patients in both groups received intravenous analgesics consisting of $1 \mathrm{~g}$ metamizole 3 times per day. Additional medication in the PIA treatment group consisted of $7.5 \mathrm{mg}$ piritramide and was administered on patient demand, monitored, and documented. Peridural catheters were removed on postoperative Day 4.

Functional outcome was measured using the trunk control test (TCT), a well-established tool for the assessment of motor deficits after stroke but may also be used after spinal surgery. This test measures 4 items (rolling to the weak side, rolling to the strong side, balancing in sitting position, sitting up from lying down), with each item scored and summarized $(0=$ unable to perform movement without assistance; $12=$ able to perform movement but in an abnormal style [pulling on bed clothes, rope, or assist pole or using arms to steady self when sitting]; $25=$ able 
to complete movement normally). For example, for the sitting balance item, a patient would score 12 if they need to touch anything with their hands to stay upright and 0 if they are unable to stay up (by any means) for 30 seconds.

Regular follow-up was scheduled for all patients. Antibiotic medication was discontinued depending on the clinical and laboratory findings, including performance in functional tests and the presence of inflammatory mediators. Calculated antibiotic treatment consisted of postoperative meropenem (6 g/day) and vancomycin ( $2 \mathrm{~g} /$ day), with any required adaptation in regard to an antibiogram, if obtained. Intravenous antibiotics were continued for 6 weeks, followed by oral antibiotics for another 6 weeks if no signs of recurrent infection were observed.

A nonparametric analysis of variance with multiple comparison adaptation was performed to compare group differences during all time points documented. Demographic data, predetermined functional targets, and the incidence of adverse events at determined end points were compared using the Mann-Whitney U-test or the $\chi^{2}$ test. Statistical analyses were performed using commercially available software (SPSS version 19, IBM). A p value less than 0.05 was considered statistically significant.

\section{Results}

Between January 2010 and June 2013, we performed surgery on 32 patients with spondylodiscitis of the thoracic and lumbar spine. According to the study design, the first 16 patients included were treated with PIA while the latter 16 patients were treated with PCEA.

Three (18.8\%) of the 16 patients assigned to the PCEA treatment group were excluded from analysis. Two patients $(66.7 \%)$ were excluded due to intraoperative damage of the dura, and 1 patient (33.3\%) had a history of drug abuse. Demographic and perioperative patient data included in the study did not differ between the study groups (Table 1).

Mean pain scores at rest for the PIA group versus the PCEA group at 4,8 , and 48 hours after surgery were 4.3 versus $2.1,3.9$ versus 2.1 , and 3.8 versus 1.6 , respectively (Fig. 1). On postoperative Days 2, 3, 4, and on discharge, the pain scores were 3.4 versus $1.5,3.1$ versus $1.5,2.9$ versus 1.6 , and 1.7 versus 1.5 , respectively. Patients assigned to the PCEA group had significantly reduced postoperative pain scores when compared with patients in the PIA group at $4(\mathrm{p}<0.0001), 8(\mathrm{p}=0.02), 24(\mathrm{p}=0.03)$, and 48 hours $(p=0.03)$, while pain levels as measured by visual analog scale (VAS) converged over time and did not display significant differences in the later time points analyzed.

Patients in the PCEA group had significantly greater trunk mobility in comparison with patients in the PIA group in performing mobilization maneuvers (Fig. 2). The TCT conducted at $8(\mathrm{p}<0.001), 24(\mathrm{p}=0.004), 48(\mathrm{p}=$ $0.015), 72(p=0.0031)$, and 96 hours $(p<0.001)$ revealed significantly greater mobilization capacity in patients of the PCEA treatment group. An exemplary analysis of trunk control capacity at postoperative Day 2 displayed a significantly lower capability of rolling to the weak side, rolling to the strong side, and balancing in sitting position in patients in the PIA group (13.7 vs $19.1, \mathrm{p}=0.03 ; 19.3$
TABLE 1. Patient demographic and operative data*

\begin{tabular}{|c|c|c|}
\hline Characteristic & $\operatorname{PIA}(n=16)$ & $\operatorname{PCEA}(n=13)$ \\
\hline Age in yrs & $62(46-82)$ & $61(20-81)$ \\
\hline $\mathrm{BMI}, \mathrm{kg} / \mathrm{m}^{2}$ & $27(17-36)$ & $27(19-35)$ \\
\hline \multicolumn{3}{|l|}{ ASA classification, $n$} \\
\hline II & 5 & 2 \\
\hline III & 9 & 11 \\
\hline IV & 2 & 0 \\
\hline Female sex, $n$ & 6 & 6 \\
\hline \multicolumn{3}{|l|}{ No. of segments affected, $n$} \\
\hline 1 & 14 & 9 \\
\hline 2 & 2 & 4 \\
\hline \multicolumn{3}{|l|}{ No. of segments fused, $n$} \\
\hline 1 & 10 & 5 \\
\hline$>1$ & 6 & 8 \\
\hline Vertebrectomy, $n$ & 2 & 4 \\
\hline \multicolumn{3}{|l|}{ Spine location, $n$} \\
\hline Thoracic/thoracolumbar & 5 & 6 \\
\hline Lumbar & 11 & 7 \\
\hline Duration of surgery, mins & $322(215-508)$ & $276(182-531)$ \\
\hline Duration of anesthesia, mins & $431(302-665)$ & $403(276-585)$ \\
\hline $\begin{array}{l}\text { Intraoperative fentanyl } \\
\text { dose, } \mathrm{mg} / \mathrm{hr}\end{array}$ & $0.18(0.08-0.7)$ & $0.18(0.1-0.68)$ \\
\hline
\end{tabular}

ASA = American Society of Anesthesiologists; $B M I=$ body mass index. * Values are mean (range) unless otherwise indicated. Data comparisons were made using the Mann-Whitney U-test and $\chi^{2}$ test. There were no statistically significant differences between the PIA and PCEA groups in any of the values analyzed.

vs $24, \mathrm{p}=0.03 ; 10.5$ vs $14, \mathrm{p}=0.04$, respectively) (Fig. 3). No significant difference was observed between groups in regard to sitting up from lying down at such an early time point after surgery ( 8.3 vs $6.5, \mathrm{p}=0.5)$.

Further analysis of trunk movement displayed significantly reduced time to normal movement in the PCEA group in all items analyzed. Moreover, abnormal turning to the weak side was achieved earlier in the PCEA group $(p<0.05)$. No significant differences between treatment groups were observed in regard to the other abnormal movements analyzed (rolling to strong side, balancing while sitting, sitting up) (Table 2).

Postoperative follow-up revealed no significant differences between the treatment groups $(\mathrm{p}=0.3)$.

Two patients $(12.5 \%)$ in the PIA treatment group and 1 patient $(8 \%)$ in the PCEA group underwent surgery for wound healing complications. One patient $(8 \%)$ in the PCEA group showed a minor extradural hematoma in relation to the catheter, which did not require intervention. No significant differences were observed in the overall occurrence of complications $(p=1)$. No cases of blood pressure depression, dural leakage as a result of catheter placement, violation of the spinal cord, allergic reactions, or meningitis were observed in the PCEA treatment group.

Pathological specimens were sent for microbiological examination. No pathogens were detected in 5 cases (31\%) of the PIA treatment group and in 4 cases $(31 \%)$ of the PCEA treatment group, yielding no statistical differ- 


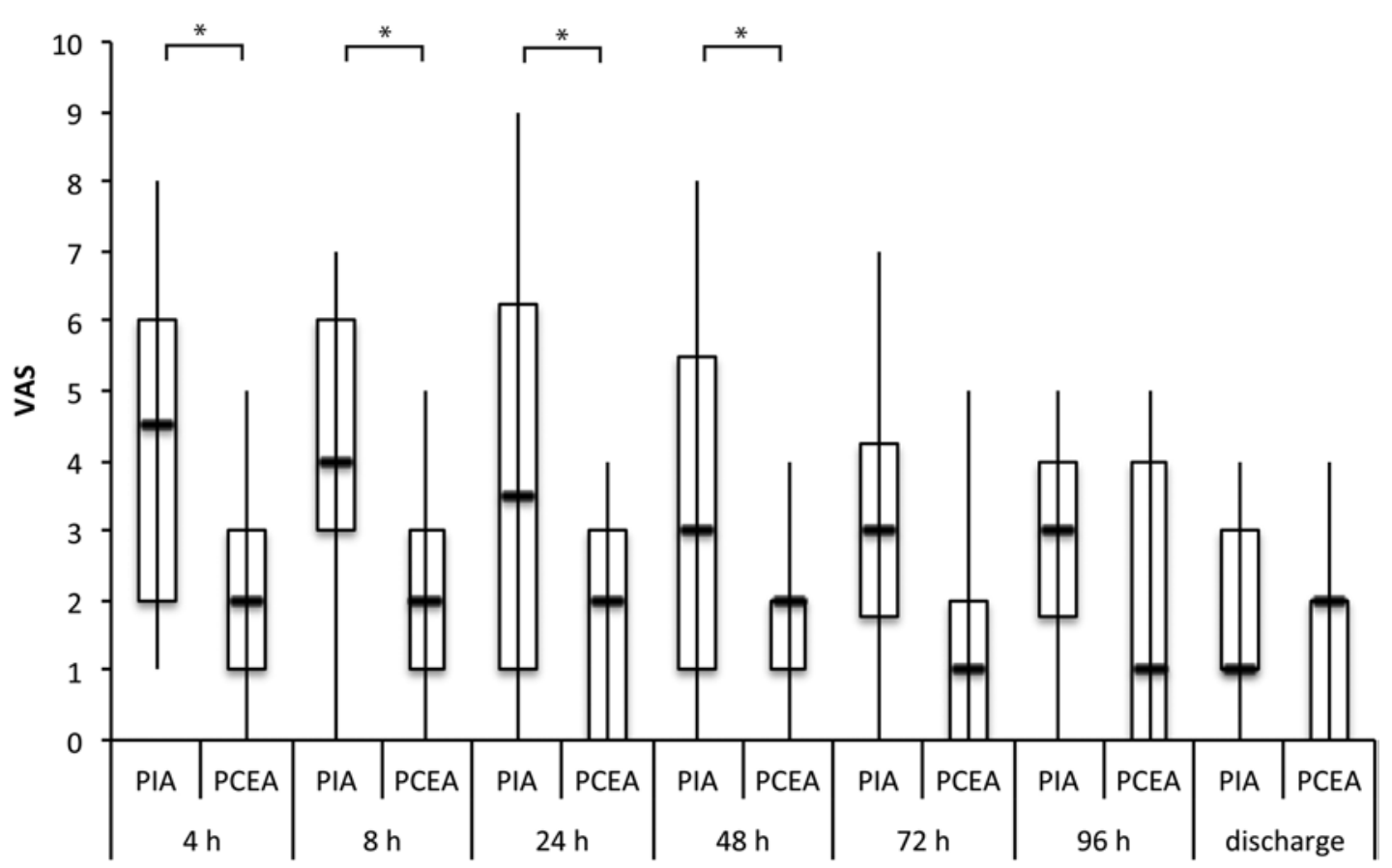

FIG. 1. Box plot showing the results of postoperative pain assessment using the VAS 4 and 8 hours after surgery, on postoperative Days 1, 2, 3, 4, and on discharge. Postoperative VAS scores after 4, 8, 24, and 48 hours are significantly reduced in the PCEA treatment group. Postoperative pain scores leveled toward discharge of patients. ${ }^{*} p<0.05$ compared with indicated treatment. The box plots show the full range (whiskers), interquartile range (boxes), and median values (horizontal rules).

ence between groups. Postoperative antibiotic treatment was conducted for at least 12 weeks and was prolonged depending on the patients' clinical examination and laboratory results. No difference was observed in overall duration of antibiotic treatment between the 2 groups. One patient in the PIA treatment group underwent a change in the antibiotic treatment due to prolonged increase of inflammatory laboratory parameters.

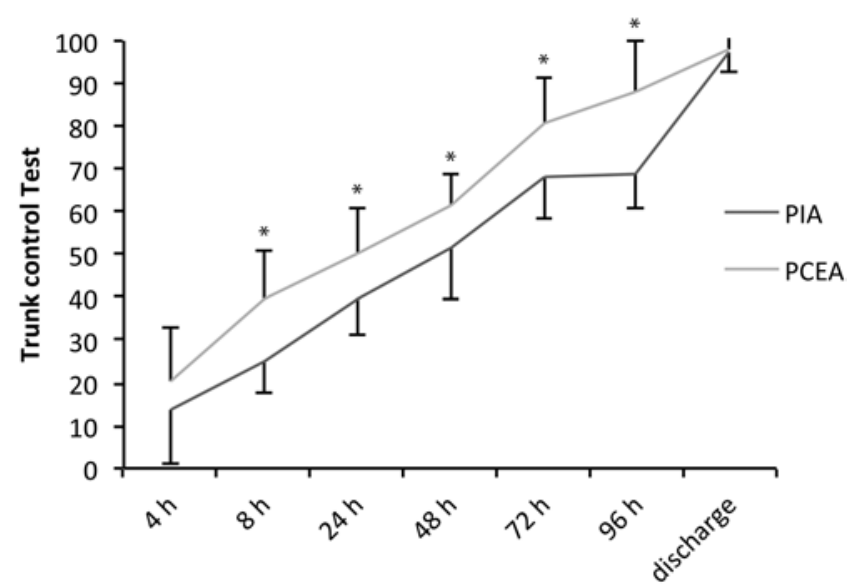

FIG. 2. Graph showing postoperative functional capacity assessed using the TCT (rolling to the weak side, rolling to the strong side, balancing in sitting position, and sitting up from lying down were observed and scored [0 = unable, $12=$ able in abnormal style, $25=$ normal style]). Patients in the PCEA group showed significantly better values for trunk movement capacity from 8 to 96 hours after surgery. No significant differences between groups were found initially after surgery (4 hours) and on discharge. ${ }^{*} p<0.05$ at indicated time point.

\section{Discussion}

To our knowledge, this is the first study challenging the dogma that PCEA is contraindicated in the presence of spinal infection by comparing safety, postoperative pain, physical capabilities, and complications after major spinal surgery in cases of spondylodiscitis based on the type of analgesia.

Analysis of the data obtained displayed normal trunk movement of all items analyzed at a significantly earlier stage in patients in the PCEA treatment group compared

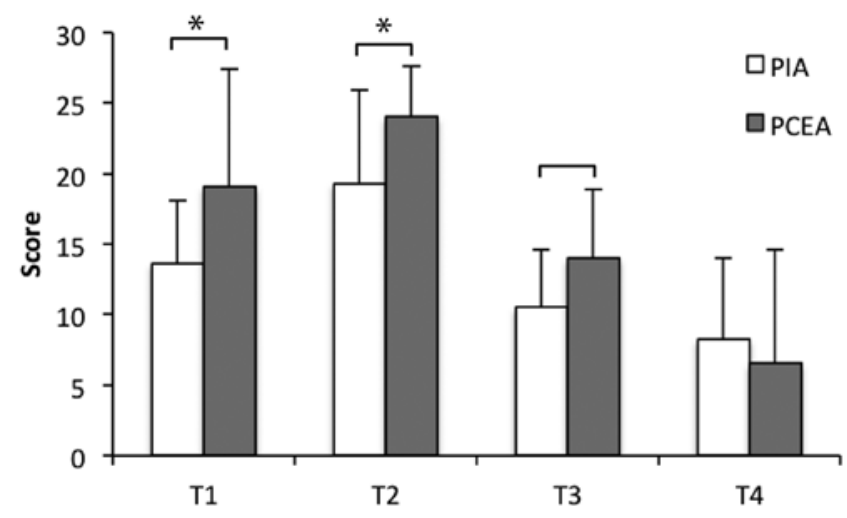

FIG. 3. Graph showing exemplary differentiation between the TCT items analyzed on postoperative Day 2. Patients in the PCEA treatment group displayed significantly better trunk movement capacities in rolling to the weak side ( $T 1, p=0.03$ ), rolling to the strong side ( $T 2, p=0.03)$, and balancing in sitting position ( $T 3, p=0.045)$. No significant differences were observed in regard to sitting up from lying down (T4, $p=0.5)$. ${ }^{*} p<$ 0.05 compared with indicated treatment. 
TABLE 2. Time to achieve defined targets of postoperative trunk mobility assessed using the TCT*

\begin{tabular}{lllc}
\hline \multicolumn{1}{c}{ Target Item } & PIA (days) & PCEA (days) & $\begin{array}{c}\text { Palue } \\
\text { Volling to weak side }\end{array}$ \\
\hline Abnormally & $0.56(0.2-2)$ & $0.21(0.1-0.3)$ & $<0.05$ \\
\hline Normally & $2.81(1-3)$ & $1.71(0.3-3)$ & $<0.01$ \\
\hline Rolling to strong side & & & \\
\hline Abnormally & $0.23(0.2-0.3)$ & $0.21(0.2-0.3)$ & $\mathrm{NS}$ \\
\hline Normally & $2.02(0.3-3)$ & $0.78(0.2-3)$ & $<0.01$ \\
\hline Balancing in sitting position & & & \\
\hline Abnormally & $1.68(0.2-5)$ & $1.17(0.2-2)$ & $\mathrm{NS}$ \\
\hline Normally & $5.31(3-7)$ & $2.69(1-3)$ & $<0.01$ \\
\hline Sitting up from lying down & & \multicolumn{2}{l}{} \\
\hline Abnormally & $2.07(1$ to $>10)$ & $2.92(1-5)$ & $\mathrm{NS}$ \\
\hline Normally & $5.92(5$ to $>10)$ & $4.45(2$ to $>10)$ & $<0.01$ \\
\hline
\end{tabular}

NS = not significant.

* Values are mean (range). Data comparisons were made using the MannWhitney U-test. Patients in the PCEA treatment group achieved normal movement in all items analyzed significantly faster than those in the PIA group. Also, rolling to the weak side in an abnormal way was achieved earlier in the PCEA treatment group $(p<0.05)$.

with patients in the PIA treatment group. These data are in line with published data of patients with posterior spinal surgery in the noninfectious setting, which demonstrated reduced time to reach turning capacities in bed in patients with epidural analgesia compared with patients with intravenous analgesia. ${ }^{16}$

In regard to technical handling of epidural catheters, no complications in the form of disruption of the dura or violation of the spinal cord were observed when visually placing the catheter, which concurs with the literature and the consensus of a more feasible intraoperative catheter insertion compared with a preoperative catheter placement (Table 3). ${ }^{7}$ Further, follow-up did not reveal any catheterassociated complications such as respiratory depression or neurological worsening, as has been previously described. ${ }^{17}$

The need for longer follow-up of patients in the PIA group may be explained by the design of the study. Overall, the rate of complications was quite high in regard to recurrent surgery for wound-healing deficits. ${ }^{5}$ This may be attributable to the relatively large number of patients in poor condition and to a high rate of comorbidities, as displayed by a relatively high American Society of Anesthesiologists physical status classification grade in this study cohort. No infectious complications directly attributable to the epidural catheter were observed. No pathogen was detected in $9(31 \%)$ of the 29 cases of spondylodiscitis, resulting in nonantibiogram-adapted calculated antibiotic therapy in those patients.

In regard to postoperative analgesia, no significant difference in the duration of postoperative antibiotic treatment was found between the treatment groups, as clinical conditions recovered well and laboratory parameters as a surrogate parameter for an infection normalized comparably over time. No significant difference in complications
TABLE 3. Patient antibiotic treatment and follow-up for clinical reevaluation*

\begin{tabular}{|c|c|c|}
\hline Characteristic & $\operatorname{PIA}(n=16)$ & $\operatorname{PCEA}(n=13)$ \\
\hline Follow-up, days & $225(8-957)$ & $140(12-505)$ \\
\hline Antibiotic regimen change, $n$ & 2 & 2 \\
\hline Complications, $n$ & 2 & 2 \\
\hline Duration of antibiotic $\mathrm{Tx}$, days & $88(8-207)$ & $89(10-505)$ \\
\hline Pathogen identification, $\mathrm{n}$ & 11 & 9 \\
\hline
\end{tabular}

resulting in any kind of consequence between the PIA and the PCEA group were observed, indicating a low-risk profile of peridural analgesia.

Apparently, combined intravenous and epidural analgesia, as administered in the PCEA group, did sufficiently decrease postoperative pain levels in these cases of spinal surgery in noninfectious cases. Higher amounts of inflammatory mediators have been described in spondylodiscitis patients. ${ }^{21}$ Postoperative analgesia by a local administration may be sufficient in these cases due to higher concentrations of the locally applied analgesic and a combination of analgesic medications.

\section{Limitations}

As this is the first series of patients with spondylodiscitis being treated with PCEA, this study has several limitations. The major flaw of this study is the lack of randomization and blinding, thus introducing a possible bias. Furthermore, studies including a larger patient population are necessary to fully evaluate the value of PCEA in patients with spondylodiscitis, especially in regard to cost effectiveness and indirect outcome parameters associated with early mobilization and impact on the development of pneumonia or pulmonary embolism.

\section{Conclusions}

The value of postoperative analgesia after major spinal surgery is unquestioned. With adequate postoperative analgesia patient recovery may be achieved earlier and to a better level of satisfaction. In this study, we have demonstrated for the first time that, even in cases of infectious spinal disorders, epidural analgesia is superior to intravenous analgesia in regard to postoperative pain and patients' mobilization maneuvers in bed. We observed neither increase in the rate of complications in patients treated with epidural analgesia nor any prolongation in postoperative signs of infection or antibiotic administration. Our data indicate that patient-controlled epidural analgesia may be administered even in patients with bacterial spinal infection following thorough debridement and instrumentation.

\section{Acknowledgments}

We would like to gratefully acknowledge the generous assistance in table editing provided by Marina Heibel. 


\section{References}

1. Acosta FL Jr, Chin CT, Quiñones-Hinojosa A, Ames CP, Weinstein PR, Chou D: Diagnosis and management of adult pyogenic osteomyelitis of the cervical spine. Neurosurg Focus 17(6):E2, 2004

2. Bianconi M, Ferraro L, Ricci R, Zanoli G, Antonelli T, Giulia $\mathrm{B}$, et al: The pharmacokinetics and efficacy of ropivacaine continuous wound instillation after spine fusion surgery. Anesth Analg 98:166-172, 2004

3. Blumenthal S, Min K, Nadig M, Borgeat A: Double epidural catheter with ropivacaine versus intravenous morphine: a comparison for postoperative analgesia after scoliosis correction surgery. Anesthesiology 102:175-180, 2005

4. Butler JS, Shelly MJ, Timlin M, Powderly WG, O'Byrne JM: Nontuberculous pyogenic spinal infection in adults: a 12-year experience from a tertiary referral center. Spine (Phila Pa 1976) 31:2695-2700, 2006

5. Cassady JF Jr, Lederhaas G, Cancel DD, Cummings RJ, Loveless EA: A randomized comparison of the effects of continuous thoracic epidural analgesia and intravenous patient-controlled analgesia after posterior spinal fusion in adolescents. Reg Anesth Pain Med 25:246-253, 2000

6. Cohen BE, Hartman MB, Wade JT, Miller JS, Gilbert R, Chapman TM: Postoperative pain control after lumbar spine fusion. Patient-controlled analgesia versus continuous epidural analgesia. Spine (Phila Pa 1976) 22:1892-1897, 1997

7. Eilers K, Schenk M, Putzier M, Volk T, Kox WJ, Zippel H: [Use of peridural catheters in the treatment of postoperative pain after spinal instrumentated fusion-an experience report.] Z Orthop Ihre Grenzgeb 140:621-625, 2002 (Ger)

8. Eysel P, Hopf C, Vogel I, Rompe JD: Primary stable anterior instrumentation or dorsoventral spondylodesis in spondylodiscitis? Results of a comparative study. Eur Spine J 6:152157,1997

9. Fisher CG, Belanger L, Gofton EG, Umedaly HS, Noonan VK, Abramson C, et al: Prospective randomized clinical trial comparing patient-controlled intravenous analgesia with patient-controlled epidural analgesia after lumbar spinal fusion. Spine (Phila Pa 1976) 28:739-743, 2003

10. Gottschalk A, Freitag M, Tank S, Burmeister MA, Kreil S, Kothe R, et al: Quality of postoperative pain using an intraoperatively placed epidural catheter after major lumbar spinal surgery. Anesthesiology 101:175-180, 2004

11. Gul H, Yildiz O, Dogrul A, Yesilyurt O, Isimer A: The interaction between IL-1 $\beta$ and morphine: possible mechanism of the deficiency of morphine-induced analgesia in diabetic mice. Pain 89:39-45, 2000

12. Kjaer P, Bendix T, Sorensen JS, Korsholm L, Leboeuf-Yde C: Are MRI-defined fat infiltrations in the multifidus muscles associated with low back pain? BMC Med 5:2, 2007

13. Klöckner C, Valencia R, Weber U: [Alignment of the sagittal profile after surgical therapy of nonspecific destructive spondylodiscitis: ventral or ventrodorsal method-a comparison of outcomes.] Orthopade 30:965-976, 2001 (Ger)

14. Morin AM, Kerwat KM, Klotz M, Niestolik R, Ruf VE, Wulf $\mathrm{H}$, et al: Risk factors for bacterial catheter colonization in regional anaesthesia. BMC Anesthesiol 5:1, 2005

15. Pao JL, Yang RS, Hsiao CH, Hsu WL: Trunk control ability after minimally invasive lumbar fusion surgery during the early postoperative phase. J Phys Ther Sci 26:1165-1171, 2014

16. Schenk MR, Putzier M, Kügler B, Tohtz S, Voigt K, Schink $\mathrm{T}$, et al: Postoperative analgesia after major spine surgery: patient-controlled epidural analgesia versus patient-controlled intravenous analgesia. Anesth Analg 103:1311-1317, 2006

17. Shaw BA, Watson TC, Merzel DI, Gerardi JA, Birek A: The safety of continuous epidural infusion for postoperative analgesia in pediatric spine surgery. J Pediatr Orthop 16:374-377, 1996

18. Sobottke R, Seifert H, Fätkenheuer G, Schmidt M, Gossmann A, Eysel P: Current diagnosis and treatment of spondylodiscitis. Dtsch Arztebl Int 105:181-187, 2008

19. Suess O, Weise L, Brock M, Kombos T: Debridement and spinal instrumentation as a single-stage procedure in bacterial spondylitis/spondylodiscitis. Zentralbl Neurochir 68:123-132, 2007

20. Tsutsumimoto T, Shimogata M, Ohta H, Misawa H: Miniopen versus conventional open posterior lumbar interbody fusion for the treatment of lumbar degenerative spondylolisthesis: comparison of paraspinal muscle damage and slip reduction. Spine (Phila Pa 1976) 34:1923-1928, 2009

21. Yoshida M, Nakamura T, Sei A, Kikuchi T, Takagi K, Matsukawa A: Intervertebral disc cells produce tumor necrosis factor alpha, interleukin-1 $\beta$, and monocyte chemoattractant protein-1 immediately after herniation: an experimental study using a new hernia model. Spine (Phila Pa 1976) 30:55-61, 2005

\section{Disclosures}

The authors report no conflict of interest concerning the materials or methods used in this study or the findings specified in this paper.

\section{Author Contributions}

Conception and design: Gessler, Weise. Acquisition of data: Gessler, Mutlak, Tizi. Analysis and interpretation of data: Gessler, Mutlak, Weise. Drafting the article: Gessler, Setzer. Critically revising the article: Gessler. Reviewed submitted version of manuscript: Gessler, Mutlak, Setzer. Approved the final version of the manuscript on behalf of all authors: Gessler. Statistical analysis: Gessler, Weise. Administrative/technical/material support: Senft, Setzer, Seifert, Weise. Study supervision: Seifert, Weise.

\section{Supplemental Information}

Previous Presentations

Portions of this paper were presented in 2013 at the Spine Section of the German Society for Neurosurgery Annual Meeting (September 27-28, Frankfurt am Main, Germany).

\section{Correspondence}

Florian Gessler, Department of Neurosurgery, University Hospital Frankfurt, Schleusenweg 2-16, D-60528 Frankfurt am Main, Germany.email: f.gessler@med.uni-frankfurt.de. 